\title{
Onion extract and quercetin induce matrix metalloproteinase-1 in vitro and in vivo
}

\author{
JAE-WE CHO ${ }^{1}$, SUN-YOUNG CHO ${ }^{1}$, SEONG-RYONG LEE ${ }^{2}$ and KYU-SUK LEE \\ Departments of ${ }^{1}$ Dermatology; ${ }^{2}$ Pharmacology, Keimyung University, \\ School of Medicine, 194 DongSan-Dong Jung-Gu, Daegu 700-712, Korea
}

Received September 23, 2009; Accepted November 5, 2009

DOI: 10.3892/ijmm_00000351

\begin{abstract}
A scar is usually developed by an imbalance of collagen synthesis and degradation. It is believed that the flavonoids (quercetin and kaempferol) in onion extract play a role in reducing scar formation through inhibition of fibroblast activities. Even though several commercial products are composed of onion extract, the precise molecular mechanisms of onion extract in reduction of scar formation in skin are still largely unknown. In this study we investigated the effect both of onion extract and quercetin on the proliferation of fibroblasts, expression of type I collagen and matrix metalloproteinase-1 (MMP-1). Our data show that proliferation rates of fibroblasts were decreased in a dose-dependent manner of the onion extract and quercetin. The expression of type I collagen was not markedly changed by the onion extract and quercetin. Interestingly, the expression of MMP-1 was markedly increased by both onion extract and quercetin in vitro and in vivo. Thus, our data indicate that onion extract and quercetin play a role in the anti-scar effect in skin through up-regulation of MMP-1 expression, implying this agent is a promising material for reducing scar formation.
\end{abstract}

\section{Introduction}

Quercetin is a flavonoid compound that occurs naturally in plants and is widely distributed in tea, vegetables and fruits such as onions, apples, and berries $(1,2)$. The flavonoid compound exerts several biological properties including antioxidant activity, tumor suppression, and reduction of scar formation (3-5). Recently, commercial products, such as Contractubex gel, which is composed of onion extract, has been used for reducing scar formations (6). However, the exact mechanism of its action is still unknown.

The abnormal growth of fibroblasts and excessive accumulation of extracellular matrix (ECM) proteins are

Correspondence to: Dr Kyu-Suk Lee, Department of Dermatology, Keimyung University School of Medicine, 194 DongSan-Dong Jung-Gu, Daegu 700-712, Korea

E-mail: janylove99@dsmc.or.kr

Key words: onion extract, quercetin, matrix metalloproteinase-1, fibroblasts prominent features of hypertrophic scar and keloid $(7,8)$. Type I collagen is a prototype of ECM and MMP-1 is a prototype of collagenase, which plays a key role in the dynamic remodeling of extracellular matrix in cells. In addition, tissue inhibitor matrix metalloproteinase-1 (TIMP-1) is an inhibitor of MMP-1 in tissue. The amount of type I collagen is tightly regulated by balance between MMP-1 and TIMP-1. Thus, if some agent induces the expression on MMP-1 in tissue, this agent is a promising candidate component of anti-scar products. In this study we examined the effects of both onion extract and quercetin on the protein levels of type 1 collagen and MMP-1 in vitro using human skin fibroblast and in vivo using hairless mice. In addition we studied the proliferation rates of onion extract-treated human skin fibroblasts.

\section{Materials and methods}

Materials. Antibodies against phospho-ERK (p-ERK), ERK, phospho-p38 (p-p38), p38, phospho-JNK (p-JNK), and JNK were purchased from Cell Signaling (Beverly, MA). Antibodies against type I collagen, MMP-1, and TIMP-1 were obtained from Santa Cruz Biotechnology (Santa Cruz, CA). Quercetin was purchased from Sigma-Aldrich (St. Louis, MO) and R\&D system (Minneapolis, MN). Onion extract was made by crushing onion and filtered with a $0.02 \mu \mathrm{m}$ disc filter.

Cell culture. Human skin fibroblasts were maintained at $37^{\circ} \mathrm{C}$ in a humidified atmosphere of $95 \%$ air and $5 \% \mathrm{CO}_{2}$ in Eagle's minimum essential medium supplemented with $10 \%$ heat inactivated fetal bovine serum (FBS), $2 \mathrm{mM}$ glutamine, and $100 \mathrm{U} / \mathrm{ml}$ penicillin and $100 \mu \mathrm{g} / \mathrm{ml}$ streptomycin. For experiments, cells $\left(5 \times 10^{4}\right.$ cells $\left./ \mathrm{ml}\right)$ were seeded in a culture dish, and maintained in the tissue culture incubator. Quercetin, ranging from 10 to $40 \mu \mathrm{M}$, was treated for $24 \mathrm{~h}$ in fibroblasts and then RNA or protein was extracted from cells.

Reverse transcription-polymerase chain reaction (RT-PCR). Total RNA was isolated from cells using the RNAzol ${ }^{\mathrm{TM}} \mathrm{B}$ (Biotecx laboratories, Houston, TX) according to the manufacturer's instructions and quantified by spectrophotometer. Total RNA ( $1 \mu \mathrm{g})$ was reverse transcribed using M-MLV reverse transcriptase (Promega Co., Madison, WI). The PCR reaction was carried out under the conditions recommended by the manufacturer (Takara Co., Otsu, Japan). Briefly, $50 \mu 1$ of a reaction mixture including 2.5 units of Taq 
polymerase (Takara Co., Otsu, Japan), $5 \mu 1$ of 10x buffer, $1.5 \mathrm{mM} \mathrm{MgCl}_{2}, 200 \mu \mathrm{M}$ dNTPs, $1 \mu \mathrm{l}$ of first-strand cDNA, and 25 pmol of each primer, was subjected to 28 PCR cycles (denaturation at $94^{\circ} \mathrm{C}$ for $1.5 \mathrm{~min}$, annealing at $58^{\circ} \mathrm{C}$ for $1 \mathrm{~min}$, and polymerization at $72^{\circ} \mathrm{C}$ for $1 \mathrm{~min}$ ). The PCR products were analyzed on $1.5 \%$ agarose gel. The primer sequences and product sizes were as follows: i) GAPDH (forward, 5'-CGT CTT CAC CAC CAT GGA GA-3', reverse, 5'-CGG CCA TCA CGC CAC AGT TT-3'), 300 base pair (bp); ii) MMP-1 (forward, 5'-ATG CAC AGC TTT CCT CCA CTG-3', reverse, 5'-CAG CCC AAA GAA TTC CTG CAT T-3'), 240 bp; iii) type I collagen (forward, 5' CTC CGG CTC CTG CTC CTC TTA 3', reverse, 5' GCA CAG CAC TCG CCC TCC C 3'), $400 \mathrm{bp}$.

Western blot analysis. Whole cell extracts were prepared in lysis buffer [10 mM Tris (pH 7.4), 5 mM EDTA, $130 \mathrm{mM}$ $\mathrm{NaCl}, 1 \%$ Triton $\mathrm{X}-100$, phenylmethylsulphonyl fluoride (PMSF, $10 \mathrm{mg} / \mathrm{ml})$, aprotinin $(10 \mathrm{mg} / \mathrm{ml})$, leupeptin $(10 \mathrm{mg} / \mathrm{ml})$, $5 \mathrm{mM}$ phenanthroline and $28 \mathrm{mM}$ benzamidine- $\mathrm{HCl}]$. For phospho-protein detection, cells were washed with ice-cold phosphate-buffered saline containing $1 \mathrm{mM} \mathrm{Na}_{3} \mathrm{VO}_{4}$ and $1 \mathrm{mM}$ $\mathrm{NaF}$, and lysed in a buffer [20 mM Tris- $\mathrm{Cl}$ (pH 8.0), $137 \mathrm{mM}$ $\mathrm{NaCl}, 10 \%$ glycerol, $1 \%$ Triton $\mathrm{X}-100,1 \mathrm{mM} \mathrm{Na} \mathrm{VO}_{4}, 1 \mathrm{mM}$ NaF, 2 mM EDTA, $200 \mathrm{nM}$ aprotinin, $20 \mu \mathrm{M}$ leupeptin, $50 \mu \mathrm{M}$ phenanthroline, $280 \mu \mathrm{M}$ benzamidine- $\mathrm{HCl}$ ]. To isolate cytosolic and nuclear proteins, cells were homogenized in ice-cold hypotonic buffer (10 mM HEPES, $10 \mathrm{mM} \mathrm{KCl,} 3 \mathrm{mM} \mathrm{MgCl}_{2}$, $0.5 \%$ NP-40, 2 mM PMSF, $1 \mathrm{mM}$ DTT, $200 \mathrm{nM}$ aprotinin) for $20 \mathrm{~min}$ and centrifuged at $12,000 \mathrm{rpm}$ for $10 \mathrm{~min}$. The supernatant was saved as a cytosolic fraction. The pellets were homogenized in ice-cold nuclear extract buffer $[10 \mathrm{mM}$ Tris-Cl (pH 7.5), 0.5 M NaCl, 2.5\% glycerol, $1.5 \mathrm{mM} \mathrm{MgCl}_{2}, 0.5 \mathrm{mM}$ EDTA, 0.5 mM EGTA, 1 mM DTT, 2 mM PMSF, $200 \mathrm{nM}$ aprotinin] for $20 \mathrm{~min}$ and centrifuged at $12,000 \mathrm{rpm}$ for $10 \mathrm{~min}$. The supernatant was saved as a nuclear fraction. The protein concentration of extracts was estimated with Bradford reagent (Bio-Rad, Hercules, CA, USA) using bovine serum albumin as the standard. Equal amounts of protein (40 $\mu \mathrm{g} /$ lane) were resolved by $10-12 \%$ sodium dodecyl sulfate-polyacrylamide gel electrophoresis, and transferred onto a nitrocellulose membrane. The membrane was then washed with Tris-buffered saline (10 mM Tris, $150 \mathrm{mM} \mathrm{NaCl}$ ) containing $0.05 \%$ Tween-20 (TBST) and blocked in TBST containing 5\% non-fat dried milk. The membrane was further incubated with respective specific antibodies such as type I collagen (1:2,000), MMP-1 (1:2,000), TIMP-1 (1:3,000), JNK and p-JNK $(1: 2,000)$, p38 and p-p38 $(1: 1,000)$, ERK and p-ERK $(1: 3,000)$, and $\beta$-tubulin $(1: 5,000)$. The membrane was continuously incubated with appropriate secondary antibodies coupled to horseradish peroxidase, and developed in the ECL Western detection reagents (Amersham Pharmacia Biotech, Piscataway, NJ, USA).

Animal study. Hairless mice SKH-1 were housed and treated according to approved animal protocols. The animals were marked on the dorsal side with a template drawing of $9 \times 9-\mathrm{mm}^{2}$ with tattoo ink for applying the agents. Ointment containing quercetin was applied to three animals and the treatment was administered 3 times per week for 2 weeks. Ultraviolet irradiation $\left(100 \mathrm{~mJ} / \mathrm{cm}^{2}\right)$ was also performed on three
(A)

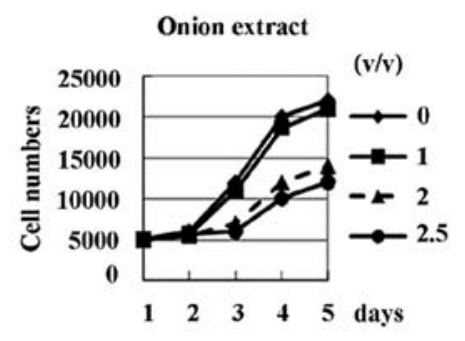

(B)

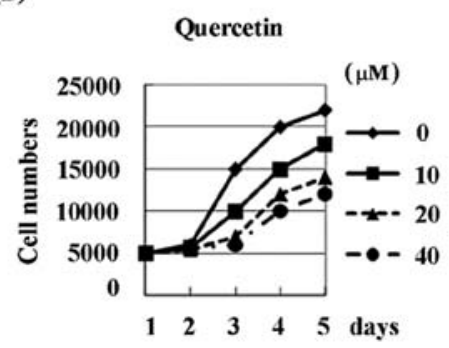

Figure 1. Growth curve of onion extract and quercetin treated-fibroblasts. Cells were cultured in the presence of onion extract (A) and quercetin (B) for 5 days. Viable cell counts were performed using trypan blue exclusion assay.

animals with or without quercetin ointment 2 times per week for 1 month. Untreated skin areas were used as controls. Animals were sacrificed one day after the last treatment.

\section{Results}

Effect of onion extract and quercetin on cell proliferation rates in human skin fibroblasts. To study the effect both of onion extract and quercetin on the proliferation rates of human skin fibroblasts, cells were exposed to onion extract $(1-2.5 \%, \mathrm{v} / \mathrm{v})$ and quercetin $(10-40 \mu \mathrm{M})$ for 7 days. As shown in Fig. 1, the proliferation rates were both decreased in a dose-dependent manner, especially in onion extract-treated human skin fibroblasts.

Effect of onion extract and quercetin on type I collagen, MMP-1 and TIMP-1 expressions in human skin fibroblasts. We analyzed the effect both of onion extract and quercetin on the expressions of MMP-1, type I collagen, and TIMP-1 in cultured fibroblasts. As shown in Fig. 2A, onion extracts induced the increased expressions of MMP-1 and type I collagen, but not the expression of TIMP-1. The increased expression of MMP-1 was clearly observed at $6 \mathrm{~h}$ after treatment (Fig. 2B). The enzymatic activity of MMP-1 was increased in onion extract-treated cultured fibroblasts by zymography using casein (data not shown). Furthermore, the slightly increased promoter activity and expression of type I collagen were confirmed by the CAT (Fig. 2C) and immunofluorescence assays (Fig. 2D), respectively. We found that the onion extract induced the increased MMP-1 expression in cultured fibroblasts, implying these molecular mechanisms explain the anti-fibrotic effect of onion extract in skin. Thus, we focused on the role of a single component of onion extract, quercetin, in MMP-1, type I collagen, and TIMP-1 expression 
(A)

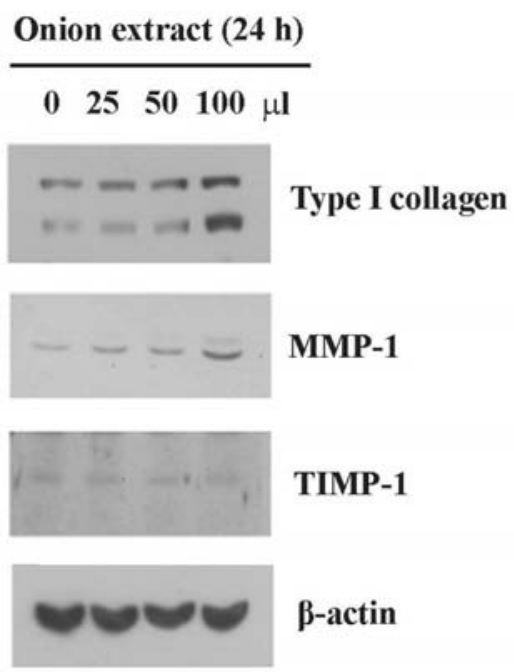

(B)

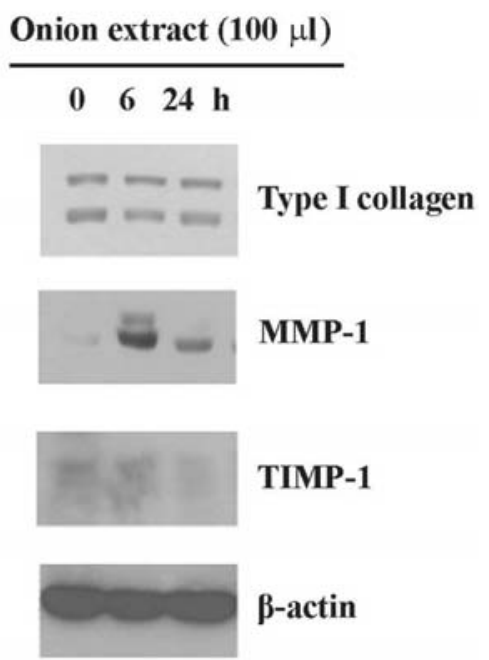

(C)

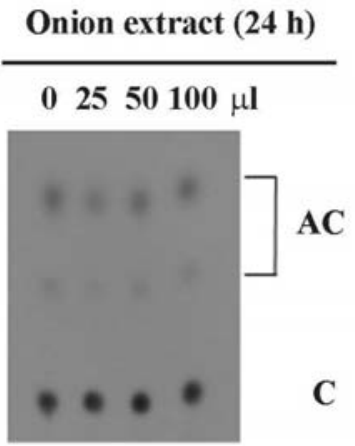

(D)

Onion extract $(100 \mu \mathrm{l})$

$(-)$

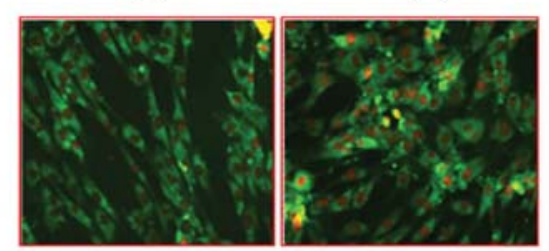

Type I collagen $(x 200)$

Figure 2. Onion extract-induced MMP-1 and type I collagen expressions. Cells were treated by onion extract and cultured for indicated times. The expressions of type I collagen, MMP-1, and TIMP-1 in fibroblasts were analyzed by Western blot analysis and immunofluorescence assay (A, B, D), and the promoter activity of type I collagen by CAT assay (C).

in cultured fibroblasts. Quercetin also markedly increased the expression of MMP-1, but not type I collagen and TIMP-1, in a dose- and time-dependent manner (Fig. 3A and B). Quercetin-induced MMP-1 expression in fibroblasts was clearly observed by immunocytochemistry using antiMMP-1 antibody (Fig. 3C). As shown in Fig. 4A and B, the expression of MMP-1 mRNA, but not type I collagen, was increased by quercetin treatment in fibroblasts, implying that quercetin-induced MMP-1 expression is partly mediated at a transcriptional level. We studied the upstream signal pathway of quercetin-induced MMP-1 expression in fibroblasts. It is well known that extracellular signal-regulated kinase (ERK), p38 mitogen activated protein kinase (MAPK), and Jun Nterminal kinase (JNK) play a role on the regulation of expression of MMP-1 under several types of stimuli. Our data showed that quercetin induced activation of JNK and ERK, but not $\mathrm{p} 38$, in cultured skin fibroblasts at $5 \mathrm{~min}$ after treatment (Fig. 4C).

Effect of quercetin ointment in ECM of hairless mouse. Finally, we studied the effect of quercetin-containing cream (0.05 and $1 \%$ ) on the expression of MMP-1 in hairless mice. We applied regularly the cream on the backs of hairless mice for 1 month, twice per week. The expression of MMP-1 was gradually increased by a dose-dependent manner (Fig. 5A). Ultraviolet B radiation induces photoaging skin which is characterized by skin atrophy and reduction of ECM. Reduction of ECM in photoaging skin is mainly mediated by MMP-1 activation. Interestingly, quercetin also plays a role as an anti-oxidant and inhibits the progression of photoaging through reducing levels of reactive oxygen species. Our data showed that quercetin markedly induced MMP-1 expression in skin fibroblasts and in vivo. Thus we examined whether the ointment containing quercetin induces the over-reduction of ECM or attenuates the reduction of ECM in UVB-irradiated hairless mice. We irradiated UVB (100 mJ) with or without ointment containing quercetin on hairless mice applied twice a week for one month and a skin biopsy was performed. The status of ECM was evaluated using Masson-Trichrome staining which is specifically deposited in collagen in dermis. As shown in Fig. 5B, markedly reduced ECM was observed in UVB irradiated hairless mice compared to control, but restoring of ECM was also observed in UVB irradiated hairless mice with quercetin ointment. Consistent with this result, the expression of MMP-1 was not markedly increased in UVB irradiated hairless mice under quercetin ointment 
(A)

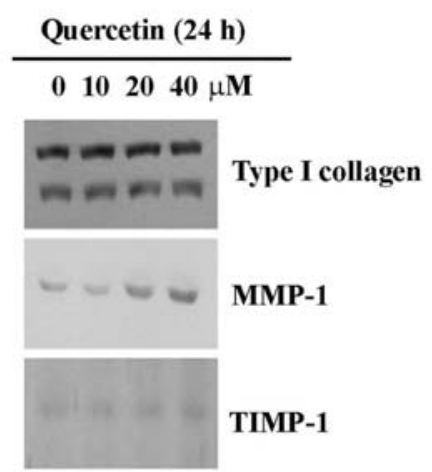

(B)

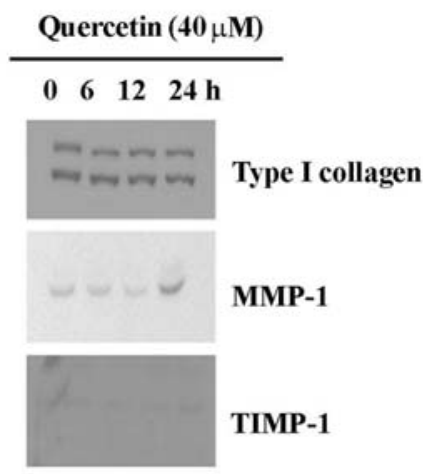

(C)

Quercetin $(40 \mu \mathrm{M})$

$(-)$

$(+)$

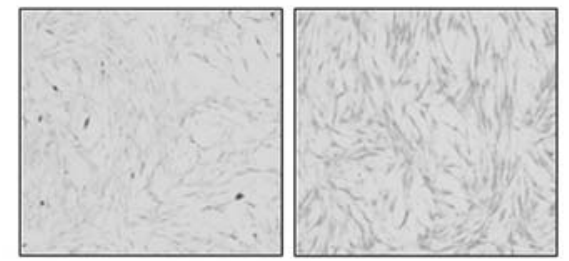

MMP-1 (x100)

Figure 3. Quercetin-induced MMP-1 expression. Cells were treated by quercetin and cultured for indicated times. The expressions of type I collagen, MMP-1, and TIMP-1 in fibroblasts were analyzed by Western blot analysis (A, B), and immunocytochemistry (C).

(A)

\begin{tabular}{llllll}
\multicolumn{4}{c}{ Quercetin $(24 \mathrm{~h})$} \\
\hline 0 & 10 & 20 & 40 & $\mu \mathrm{M}$
\end{tabular}

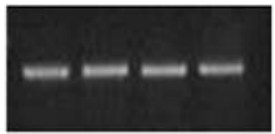

GAPDH

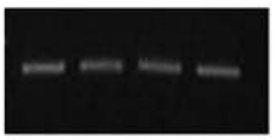

Type I collagen

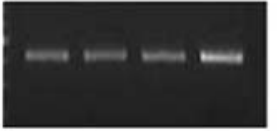

MMP-1
(B)

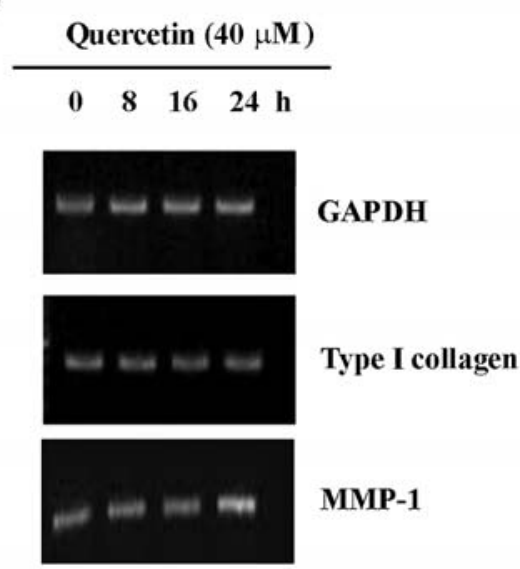

Figure 4. Up-regulation of MMP-1 mRNA and activation of JNK and ERK. Cells were treated by quercetin and cultured for indicated concentrations (A) and times (B). The expressions of MMP-1 and type I collagen mRNA were analyzed by RT-PCR analysis. Two independent experiments showed similar results. Activation of JNK and ERK were detected by anti-JNK and anti-ERK antibodies (C).

(C)

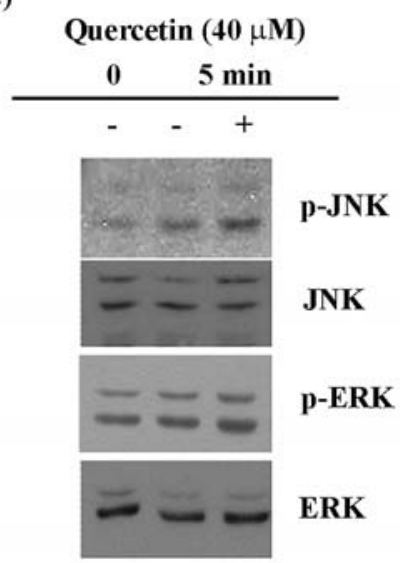


(A)

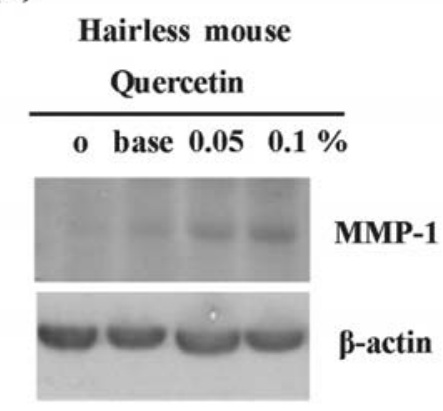

(C)

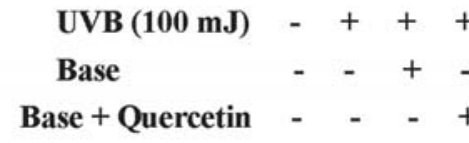

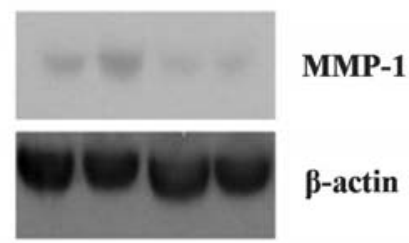

and base, compared to UVB irradiated mice (Fig. 5C). Taken together, even though quercetin and UVB itself induced the MMP-1 expression in cells, ECM was not markedly reduced by combination of quercetin and UVB, implying that quercetin also plays a role in attenuating the UVB-induced ECM reduction in tissues.

\section{Discussion}

It is well known that the expression of matrix metalloproteinase (MMP)-1 induces the degradation of extracelluar matrix (ECM) components, including type I collagen during the wound healing process (9). MMPs are able to cleave components of cell-cell junctions and cell-matrix contacts within the epithelium to promote re-epithelialization. MMP-1 is present in human cutaneous wounds during re-epithelialization but turns off once wound closure is completed $(10,11)$. Alteration of ECM is integral to the resolution of wound healing, thereby resulting in reducing scar formation. Thus, MMPs are key regulators of multiple aspects of tissue repair.

If imbalance of MMP-1 activity occurs between ECM syntheses during the wound healing process, the excessive accumulation of extracellular matrix may result in the formation of hypertrophic scar or keloid formation. Both pathologic conditions may be mediated by excessive accumulation of type I collagen, decreased activity of MMP-1, and increased expression of TIMP-1. Onion extract has been shown to exert fibroblast inhibiting properties in several studies (12). It is believed that the flavonoids (quercetin and kaempferol) in onion extract play a role in fibroblast inhibition and other anti-
(B)

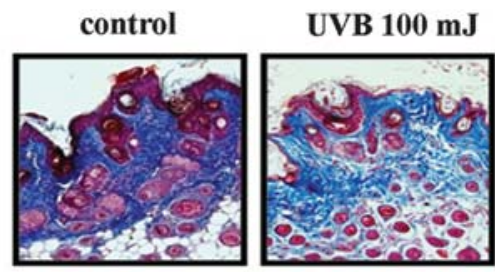

UVB $100 \mathrm{~mJ}$

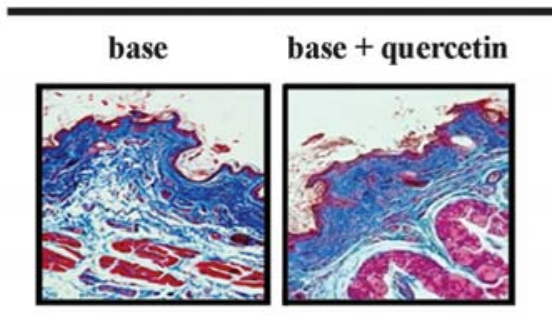

Figure 5. Induction of MMP-1 expression in quercetin-treated hairless mice. Ointment containing quercetin $(\mathrm{w} / \mathrm{o}, 0.05 \%, 0.1 \%)$ was applied on hairless mice twice a week for 1 month and then tissue extract was subjected to Western blot analysis using anti-MMP1 antibody (A). In addition UVB was irradiated for 1 month (2/weeks) with or without quercetin ointment and then the tissues were stained using Masson-Trichrome (B). In addition proteins were extracted from tissues and then subjected to Western blot analysis using anti-MMP-1 antibody (C)

proliferative effects. Several commercial products are composed of onion extract for reducing scar formation and promotion of the wound healing processes (6). However, it is still largely unknown how onion extract reduces the scar formation in skin. In addition, it is unclear whether onion extract induces the expression of MMP-1 in vivo and in vitro. Our data clearly showed that onion extract and quercetin induced the up-regulation of MMP-1 expression in human skin fibroblasts and in hairless mice, implying that onion extract and quercetin play a role in anti-fibrotic processes. Interestingly, flavonoids including quercetin showed the inhibition of ultraviolet (UV) induced-MMP expression in cells (13), implying that quercetin exerts anti-oxidant activities in the presence of UV. In addition our data indirectly showed the anti-oxidant property of quercetin in the presence of UVB, which is supported by restoring of ECM in UVB irradiated hairless mouse. Even though this result seems to make the role of onion extract on anti-fibrotic processes confusing, onion extract and quercetin itself can induce the modification of ECM through up-regulation of MMP-1.

Furthermore the biological role of MMPs is not confined to their ability to degrade the extracellular matrix. They also participate in the regulation of cellular processes such as differentiation, proliferation, angiogenesis, migration, invasion and apoptosis by interacting with growth factors, cytokines, integrins and cell surface receptors (14), thereby ointment containing quercetin is a promising wound healing promoting as well as anti-fibrosis agent.

Extracellular signal-regulated kinase (ERK), p38 mitogen activated protein kinase (MAPK), and Jun N-terminal kinase 
(JNK) play a role on the regulation of MMP-1 expression under biological stimulus (15-18). UVB-induced MMP-1 expression was mediated by ERK and JNK signaling pathways. In addition Yang et al reported that trans-zeatin inhibited UVB-induced MMP-1 expression via MAP kinases signaling in human skin fibroblasts (18). Similar to this result, we also observed that the activation of JNK and ERK, but not p38, in quercetin-treated fibroblasts, were followed by MMP-1 up-regulation.

Taken together our data suggest that onion extract and quercetin play a role in the anti-fibrotic effect and promotion of wound healing in skin through up-regulation of MMP-1 expression. Further studies are required to prove the precise molecular mechanisms of quercetin-induced promotion of wound healing or reduction of scar formation in vivo.

\section{Acknowledgements}

This work was supported by a research promoting grant from the Keimyung University Dongsan Medical Center in 2007.

\section{References}

1. Ross JA and Kasum CM: Dietary flavonoids: bioavailability, metabolic effects, and safety. Annu Rev Nutr 22: 19-34, 2002.

2. Arai Y, Watanabe S, Kimira M, Shimoi K, Mochizuki R and Kinae N: Dietary intakes of flavonols, flavones and isoflavones by Japanese women and the inverse correlation between quercetin intake and plasma LDL cholesterol concentration. J Nutr 30: 2243-2250, 2000.

3. Saric A, Balog T, Sobocanec S, et al: Antioxidant effects of flavonoid from Croatian Cystus incanus L. rich bee pollen. Food Chem Toxicol 47: 547-554, 2009.

4. Kandaswami C, Lee LT, Lee PP, Hwang JJ, Ke FC, Huang YT and Lee MT: The antitumor activities of flavonoids. In Vivo 19: 895-909, 2005

5. Hosnuter M, Payasli C, Isikdemir A and Tekerekoglu B: The effects of onion extract on hypertrophic and keloid scars. J Wound Care 16: 251-254, 2007.

6. Ho WS, Ying SY, Chan PC and Chan HH: Use of onion extract, heparin, allantoin gel in prevention of scarring in Chinese patients having laser removal of tattoos: a prospective randomized controlled trial. Dermatol Surg 32: 891-896, 2006.
7. Bran GM, Goessler UR, Hormann K, Riedel F and Sadick H: Keloids: Current concepts of pathogenesis (Review). Int J Mol Med 24: 283-293, 2009.

8. Seifert $\mathrm{O}$ and Mrowietz U: Keloid scarring: bench and bedside. Arch Dermatol Res 301: 259-272, 2009.

9. Gill SE and Parks WC: Metalloproteinases and their inhibitors: regulators of wound healing. Int J Biochem Cell Biol 40: 1334-1337, 2008.

10. Peled ZM, Phelps ED, Updlike DL, et al: Matrix metalloproteinases and the ontogeny of scarless repair: the other side of the wound healing balance. Plast Reconstr Surg 110: 801-811, 2002.

11. Gu Q, Wang D, Gao Y, et al: Expression of MMP1 in surgical and radiation-impaired wound healing and its effects on the healing process. J Environ Pathol Toxicol Oncol 21: 71-78, 2002.

12. Majewski S and Chadzynska M: Effects of heparin, allantoin and cepae extract on the proliferation of keloid fibroblasts and other cells in vitro. Dermatol Monatsschr 174: 106-129, 1988.

13. Moon SK, Cho GO, Jung SY, et al: Quercetin exerts multiple inhibitory effects on vascular smooth muscle cells: role of ERK1/2, cell-cycle regulation, and matrix metalloproteinase-9. Biochem Biophys Res Commun 301: 1069-1078, 2003.

14. Leeman MF, Curran S and Murray GI: New insights into the roles of matrix metalloproteinases in colorectal cancer development and progression. J Pathol 201: 528-534, 2003.

15. Moon HI, Lee JG, Kwak JH and Chung JH: Isoflavonoid from Viola hondoensis, regulates the expression of matrix metalloproteinase-1 in human skin fibroblasts. Biol Pharm Bull 28: 925-928, 2005.

16. Westermarck J, Holmstrom T, Ahonen T, et al: Enhancement of fibroblast collagenase-1 (MMP-I) gene expression by tumor promoter okadaic acid is mediated by stress-activated protein kinases Jun N-terminal kinase and p38. Matrix Biol 17: 547$557,1998$.

17. Moon HJ, Lee SR, Shin SN, et al: Fucoidan inhibits UVBinduced MMP-1 expression in human skin fibroblasts. Biol Pharm Bull 31: 284-289, 2008.

18. Yang B, Ji C, Chen W, Bi Z and Wan Y: Trans-zeatin inhibits UVB-induced matrix metalloproteinase-1 expression via MAP kinases signaling in human skin fibroblasts. Int J Mol Med 23: 555-560, 2009. 